\title{
Maturation of the immune system and immunodeficiency
}

\author{
J. J. T. OWEN
}

From the Department of Anatomy, University of Birmingham, Birmingham

I will concentrate on recent work that has improved our understanding of the earliest phases of lymphocyte maturation, since this work provides a basis for the analysis of various forms of immunodeficiency. However, the reasons for interest in primary lymphocyte differentiation extend beyond a consideration of immunodeficiency. According to the clonal selection theory, which has received considerable support from experimental work, the broad range of foreign antigens which may enter the body are recognised by a repertoire of lymphocytes, each specific for a particular antigen. There is evidence that this repertoire of lymphocytes is acquired during the primary differentiation of lymphocytes that occurs in ontogeny. In addition, although the mechanisms of tolerance to 'self' antigens are poorly understood studies on lymphocyte ontogeny may provide important clues.

Lymphocytes, like other blood cells, belong to a renewal system and production of new lymphocytes is not confined to ontogeny. Hence, in discussing the primary differentiation of lymphocytes, sites of lymphocyte production in the adult must also be considered.

\section{B lymphocyte differentiation}

\section{SITES OF FORMATION}

Although the bursa of Fabricius has been known to be the site of primary B lymphocyte differentiation in birds for some years (Lydyard et al., 1976), the sites of $B$ cell production in mammals have been poorly defined. A characteristic feature of B lymphocytes is the presence of readily detectable surface immunoglobulin (sIg) which is thought to act as an antigen-recognition receptor. B lymphocytes identified in this way have been demonstrated in various sites of fetal haemopoiesis such as spleen, liver, and bone marrow. Furthermore, B lymphocytes can be generated in each of these organs in vitro (Owen et al., 1975). Thus B lymphocyte differentiation in the embryo takes place in sites of general haemopoiesis, fetal liver being the main site of production. B lymphocytes in the adult are generated in the bone marrow (Osmond and Nossal, 1974).

\section{PRE-B CELLS}

B lymphocytes are detected in human fetal liver for the first time at 9 weeks' gestation. These cells have surface IgM (sIgM). Two weeks prior to the appearance of B cells, however, large lymphoid cells containing low amounts of intracytoplasmic IgM are found in fetal liver (Gathings et al., 1977). It is thought that these cells are precursors of B lymphocytes, and they have been named pre-B cells. Pre-B cells are found in adult bone marrow, as might be expected in view of its role in B cell generation. B cells in the mouse embryo are first found in fetal liver and spleen at 16 days' gestation, and pre-B cells are found as early as 11 days' gestation (Raff et al., 1976). Pre-B cells are heterogeneous in size, ranging from large lymphoid cells to small lymphocytes, and are in active cell cycle (Owen et al., 1977). They synthesise 7s IgM of rapid turnover (Melchers et al., 1976). Thus newly-formed B lymphocytes with sIgM are derived from a proliferating population of pre-B cells which synthesise rapid-turnover intracytoplasmic IgM. In turn, pre-B cells are known to be derived from pluripotential haemopoietic stem cells-that is, stem cells with potentialities for differentiation into all of the blood cell types (Phillips et al., 1977).

This scheme of B lymphocyte maturation is relevant to the analysis of immunodeficiency, since recently it has been shown that some patients with hypogammaglobulinaemia lack B lymphocytes as well as plasma cells. This group includes boys with infantile X-linked agammaglobulinaemia, some patients having immunodeficiency with thymoma and some with varied immunodeficiency (Cooper $e t$ al., 1971; Preud'homme et al., 1973). In further studies a number of patients with infantile X-linked 
agammaglobulinaemia who lacked B lymphocytes were found to have a normal bone marrow frequency of pre-B cells (Vogler et al., 1976). Thus maturation defects at various points in the $B$ lymphocyte differentiation pathway may be encountered.

\section{NEWLY FORMED B CELLS}

As mentioned earlier, there is evidence that the repertoire of lymphocytes is present during ontogeny. Indeed, it is claimed that diversity of antigen recognition receptors is manifest as soon as lymphocytes with sIg are generated (D'Estachio and Edelman, 1975). For a number of reasons, however, this does not imply that embryos are fully capable of immune responses. Firstly, other cell types such as $T$ lymphocytes and macrophages, which are required for a response, may be too immature to participate. Secondly, newly formed B lymphocytes react differently from mature B cells when they encounter antigen. Under certain experimental conditions newly formed cells are rendered unresponsive (that is, tolerant) to antigen whereas, under the same circumstances, mature $B$ cells respond by differentiation to antibody-secreting cells (Metcalf and Klinman, 1976). In parallel with this observation, newly-formed B cells cease to synthesise cell surface Ig when they are incubated with anti-Ig antibodies, whereas mature B cells continue to do so (Raff et al., 1975). This form of receptor inactivation may provide a model for the antigen-induced tolerance of new B cells, which in turn may be one of the ways by which tolerance to 'self' antigens is achieved.

The immaturity of newly formed B cells is also shown by the fact that they lack receptors for complement and express only sIgM. The mechanisms by which various isotypes are expressed on B cells has been the subject of considerable recent debate. The picture is complex because it has now been shown that multiple isotypes may be present on individual B cells. Thus cells which were originally sIgM-positive may express $\operatorname{sIgG}$ and $\operatorname{sIgD}$ at the same time (Lawton et al., 1977). The presence of sIgD on many cells is puzzling because of the low levels of serum IgD. It is thought that SIgD may have a special role, probably in the triggering of B cells (Vitetta and Uhr, 1977).

To summarise, B lymphocytes are generated in sites of haemopoiesis along with other blood cell types. The first B lymphocytes to be formed carry sIgM although they are preceded by pre-B cells which have low amounts of cytoplasmic IgM. In turn, these cells are derived from pluripotential haemopoietic stem cells, but the precise relationship between the two is unclear. Newly formed B lymphocytes have specificity for antigen but they are more readily rendered tolerant by antigen than is the case for mature cells found in the adult. Multiple isotypes may be expressed on individual $B$ cells.

Finally, the factors controlling the maturation of B lymphocytes are poorly understood. It is known that both hormones and so-called microenvironmental influences operate in the production of blood cells, but the extent to which they also operate in the maturation of B lymphocytes is unknown. Recent studies on cultures of bone marrow have indicated that it is possible to obtain the maturation of certain blood cell types and the renewal of stem cells provided that appropriate stromal cells are present (Schrader and Schrader, 1978). However, these cultures have not led as yet to the production of $B$ lymphocytes.

\section{T lymphocyte differentiation}

\section{COMPONENTS OF THE THYMUS}

There is little doubt that the thymus plays a central role in the generation of $\mathrm{T}$ lymphocytes. The thymus itself is derived embryologically from a number of different sources. The major component of the organ is derived from the epithelium of the pharyngeal pouches, but the mesenchyme of the pharyngeal region, which is in turn derived from neural crest, contributes to the structure of the organ (Le Douarin and Jotereau, 1975). But the lymphocytes are know $\Phi$ to be derived from cells that have a quite separate origin. Migrant stem cells enter the thymus from the blood stream at an early stage of embryogenesis and under the influence of the thymus stroma they proliferate and differentiate into the diverse T lymphocyte subsets (Moore and Owen, 1967b).

\section{PRE-T CELLS}

Although the precursors of $\mathrm{T}$ lymphocytes (pre-T cells) are known to enter the thymus from the blood stream their initial sites of origin and nature are not fully understood. The various haemopoietic sites of the embryo and the adult are known to contain precursors of $T$ lymphocytes which are capable of migration to the thymus, and it is assumed that these are the normal sites of origin (Basch and Kadish, 1977). Although T lymphocytes, like B lymphocytes, are ultimately derived from a common stem cell type (pluripotential stem cells) (Abramson et al., 1977) the nature of the cell which migrates to the thymus has been the subject of controversy and speculation. One possibility is that the migrant cells are themselves pluripotential stem cells (Moore and Owen, 1967a). However, a number of workers have suggested that the migrants are already differentiated along the $T$ cell pathway. The evidence for this notion is indirect and based mainly on claims that the precursor cells, as they reside in haemopoietic 
sites before migration to the thymus, express low amounts of $\mathrm{T}$ cell surface antigens (Roelants et al., 1976) or can be induced to express these antigens by treatment with a variety of agents (including putative thymic hormones) which are known to raise intracellular cyclic AMP levels (Komuro and Boyse, 1973).

Much depends on whether these cells really are precursors of $\mathrm{T}$ lymphocytes which will migrate to the thymus. In the studies performed to date this has not been shown directly. Therefore the question must remain open whether pre-T cells are already committed to $\mathrm{T}$ cell differentiation or whether they are pluripotential haemopoietic stem cells which differentiate into $T$ cells within the environment of the thymus. A further intriguing question concerns the nature of the attractiveness of the thymic epithelium for migrant stem cells. It is known that there is a wave of migration of stem cells into the thymus early in embryogenesis and subsequently only a slow inflow which continues into adult life. It has been postulated that chemotactic factors may be released from the epithelium which draw in migrant cells at the appropriate time (Jotereau and Houssaint, 1977).

\section{THYMUS LYMPHOCYTES}

Once within the thymus the stem cells proliferate and give rise to large numbers of small lymphoid cells. Many of these cells appear immature by most functional tests and only a minority of the total thymus lymphocyte population reacts in various immunological assays - that is, mixed lymphocyte cultures, mitogen responsiveness, etc. Thus, although functional subsets of $T$ lymphocytes are present within the thymus, the pathway by which they mature is largely unknown (Shortman, 1977). One possibility is that the mature cells are derived from the immature population. There is some evidence, however, that the mature cells may be derived by a separate pathway of maturation that does not include the immature cell type. It is also unclear whether only the mature cells migrate to peripheral lymphoid organs or whether some of the immature cells themselves migrate to lymphoid organs, where they may mature further.

\section{THYMUS HORMONES}

There is considerable interest in the nature of the factors responsible for the maturation of $T$ lymphocytes within the thymus. A number of thymus hormones have been described (reviewed in Bach, 1976), but satisfactory bioassays for these hormones have been difficult to establish. Certainly none of these hormones can replace normal thymus function in immunodeficient animals, and their physiological significance must remain in some doubt. Possibly the controlling factors operate within the thymus either by short-range substances or through cell-to-cell contact. Another point of considerable interest is the way in which $T$ lymphocytes learn to recognise the histocompatibility type of the thymus epithelium as 'self' (Zinkernagel et al., 1978). This is important because of the evidence that $\mathrm{T}$ lymphocytes recognise antigen in association with 'self' histocompatibility antigen.

In summary, $\mathrm{T}$ lymphocytes are generated within the thymus from precursors which migrate into it from the blood stream. Although these pre-T cells are present in haemopoietic organs their nature is largely unknown, and it is unclear whether they are pluripotential haemopoietic stem cells or whether they are 'committed' to the T lymphocyte maturation pathway before entry to the thymus. Within the thymus stem cells mature into the various $T$ cell subsets. However, the factors controlling this maturation process and the way in which the functional subsets are derived are unknown. Although thymic hormones may be operative in this process, convincing evidence of their physiological importance is as yet lacking. Clearly, defects in $\mathrm{T}$ cell maturation may occur at many points in the differentiation pathway and may involve cells of the thymic stroma as well as the lymphoid lineage.

\section{References}

Abramson, G., Miller, R. G., and Phillips, R. A. (1977). The identification in adult bone marrow of pluripotent and restricted stem cells of the myeloid and lymphoid systems. Journal of Experimental Medicine, 145, 15671579.

Bach, J. F. (1976). The mode of action of thymic hormones and its relevance to $\mathrm{T}$-cell differentiation. Transplantation Proceedings, 8, 243-248.

Basch, R. S., and Kadish, J. L. (1977). Hematopoietic thymocyte precursors. II. Properties of the precursors. Journal of Experimental Medicine, 145, 405-419.

Cooper, M. D., Lawton, A. R., and Bockman, D. E. (1971). Agammaglobulinaemia with B lymphocytes: specific defect of plasma-cell differentiation. Lancet, 2 , 791-795.

D'Estachio, P., and Edelman, G. M. (1975). Frequency and activity of specific antigen-binding cells in developing mice. Journal of Experimental Medicine, 142, 1078-1091.

Gathings, W. E., Lawton, A. R., and Cooper, M. D. (1977). Immunofluorescent studies of the development of pre-B cells, B lymphocytes and immunoglobulin isotype diversity in humans. European Journal of Immunology, 7, 804-810.

Jotereau, F. V., and Houssant, E. (1977). Experimental studies on the migration and differentiation of primary lymphoid stem cells in the avian embryo. Developmental Immunobiology, edited by J. B. Solomon and J. D. 
Horton, pp. 123-130. North Holland, Amsterdam.

Kumuro, K., and Boyse, E. A. (1973). In-vitro demonstration of thymic hormone in the mouse by conversion of precursor cells into lymphocytes. Lancet, 1, 740-743.

Lawton, A. R., Kearney, J. F., and Cooper, M. D. (1977). Control of expression of $\mathrm{C}$ region genes during development of B cells. In Progress in Immunology III, edited by T. E. Mandel et al., pp. 171-182. North Holland, Amsterdam.

Le Douarin, N. M., and Jotereau, F. V. (1975). Tracing of cells of the avian thymus through embryonic life in interspecific chimeras. Journal of Experimental Medicine, 142, 17-40.

Lydyard, P. M., Grossi, C. E., and Cooper, M. D. (1976). Ontogeny of $B$ cells in the chicken. 1. Sequential development of clonal diversity in the bursa. Journal of Experimental Medicine, 144, 79-97.

Melchers, F., Cone, R. E., Von Boehmer, H., and Sprent, J. (1976). Immunoglobulin turnover in B lymphocyte subpopulations. European Journal of Immunology, 5, 382-388.

Metcalf, E. S., and Klinman, N. R. (1976). In vitro tolerance induction of neonatal murine B cells. Journal of Experimental Medicine, 143, 1327-1340.

Moore, M. A. S., and Owen, J. J. T. (1967a). Stem-cell migration in developing myeloid and lymphoid systems. Lancet, 2, 658-659.

Moore, M. A. S., and Owen, J. J. T. (1967b). Experimental studies on the development of the thymus. Journal of Experimental Medicine, 126, 715-725.

Osmond, D. G., and Nossal, G. J. V. (1974). Differentiation of lymphocytes in mouse bone marrow. II. Kinetics of maturation and renewal of antiglobulinbinding cells studied by double labeling. Cellular Immunology, 13, 132-145.

Owen, J. J. T., Raff, M. C., and Cooper, M. D. (1975). Studies on the generation of B lymphocytes in the mouse embryo. European Journal of Immunology, 5, 468-473.

Owen, J. J. T., Wright, D. E., Habu, S., Raff, M. C., and Cooper, M. D. (1977). Studies on the generation of B lymphocytes in fetal liver and bone marrow. Journal of Immunology, 118, 2067-2072.
Phillips, R. A., Melchers, F., and Miller, R. G. (1977). Stem cells and the ontogeny of B lymphocytes. In Progress in Immunology III, edited by T. E. Mandel et al., pp. 155-161. North Holland, Amsterdam.

Preud'homme, J. L., Griscelli, C., and Seligmann, M. (1973). Immunoglobulins on the surface of lymphocytes in fifty patients with primary immunodeficiency diseases. Clinical Immunology and Immunopathology, 1, 241-256.

Raff, M. C., Megson, M., Owen, J. J. T., and Cooper, M. D. (1976). Early production of intracellular IgM by B-lymphocyte precursors in mouse. Nature (London), 259, 224-226.

Raff, M. C., Owen, J. J. T., Cooper, M. D., Lawton, A. R., III, Megson, M., and Gathings, W. E. (1975). Differences in susceptibility of mature and immature mouse B lymphocytes to anti-immunoglobulin-induced immunoglobulin suppression in vitro: possible implications for B-cell tolerance to self. Journal of Experimental Medicine, 142, 1052-1064.

Roelants, G. E., Mayor, K. S., Hägg, L. B., and Loor, F. (1976). Immature T lineage lymphocytes in athymic mice. Presence of TL, lifespan and homeostatic regulation. European Journal of Immunology, 6, 75-81.

Schrader, J. W., and Schrader, S. (1978). In vitro studies on lymphocyte differentiation. I. Long term in vitro culture of cells giving rise to functional lymphocytes in irradiated mice. Journal of Experimental Medicine, 148, 823-828.

Shortman, K. (1977). The pathway of T cell development within the thymus. In Progress in Immunology III, edited by T. E. Mandel et al., pp. 197-205. North Holland, Amsterdam.

Vitetta, E. S., and Uhr, J. W. (1977). IgD and B cell differentiation. Inemunological Reviews, 37, 50-88.

Vogler, L. B., Pearl, E. R., Gathings, W. E., Lawton, A. R., and Cooper, M. D. (1976). B lymphocyte precursors in bone-marrow in immunoglobulin deficiency diseases (Letter). Lancet, 2, 376.

Zinkernagel, R. M., Callahan, G. N., Klein, J., and Dennert, G. (1978). Cytotoxic T cells learn specificity for self $\mathbf{H}-2$ during differentiation in the thymus. Nature (London), 271, 251-253. 